\title{
Confidence in beliefs about pain predicts expectancy effects on pain perception and anticipatory processing in right anterior insula
}

\author{
Christopher A. Brown ${ }^{\mathrm{a}, *}$, Ben Seymour ${ }^{\mathrm{b}}$, Wael El-Deredy ${ }^{\mathrm{c}}$, Anthony K.P. Jones ${ }^{\mathrm{a}}$ \\ ${ }^{a}$ Human Pain Research Group, Clinical Sciences Building, Hope Hospital, Salford M6 8HD, United Kingdom \\ ${ }^{\mathrm{b}}$ Wellcome Department of Imaging Neuroscience, Functional Imaging Laboratory, 12 Queen Square, London WC1N 3BG, United Kingdom \\ ${ }^{\mathrm{c}}$ School of Psychological Sciences, University of Manchester, Zochonis Building, Oxford Road, Manchester M13 9PL, United Kingdom
}

Received 27 February 2008; received in revised form 24 April 2008; accepted 30 April 2008

\begin{abstract}
Psychological factors play a major role in exacerbating chronic pain. Effective self-management of pain is often hindered by inaccurate beliefs about the nature of pain which lead to a high degree of emotional reactivity. Probabilistic models of perception state that greater confidence (certainty) in beliefs increases their influence on perception and behavior. In this study, we treat confidence as a metacognitive process dissociable from the content of belief. We hypothesized that confidence is associated with anticipatory activation of areas of the pain matrix involved with top-down modulation of pain. Healthy volunteers rated their beliefs about the emotional distress that experimental pain would cause, and separately rated their level of confidence in this belief. Confidence predicted the influence of anticipation cues on experienced pain. We measured brain activity during anticipation of pain using high-density EEG and used electromagnetic tomography to determine neural substrates of this effect. Confidence correlated with activity in right anterior insula, posterior midcingulate and inferior parietal cortices during the anticipation of pain. Activity in the right anterior insula predicted a greater influence of anticipation cues on pain perception, whereas activity in right inferior parietal cortex predicted a decreased influence of anticipatory cues. The results support probabilistic models of pain perception and suggest that confidence in beliefs is an important determinant of expectancy effects on pain perception.
\end{abstract}

(C) 2008 International Association for the Study of Pain. Published by Elsevier B.V. All rights reserved.

Keywords: Anticipation; Expectancy; Nociception; Physiology; Prediction

\section{Introduction}

Psychological influences on chronic pain, such as negative expectations and beliefs, can increase the perception of pain by potentiating emotional reactivity [48]. The research focus is now on understanding the neural mechanisms by which expectations, beliefs and conditioning may act to influence the experience of pain. Brain networks involved with aversive conditioning have been investigated in humans and other animals $[17,26]$. Furthermore, human neuroimaging studies have

\footnotetext{
${ }^{*}$ Corresponding author. Tel.: +44 1612064528.

E-mail address: Christopher.Brown@Manchester.ac.uk (C.A. Brown).
}

elucidated key areas of the brain associated with the robust effect of expectations on the perception of pain using methodologies that manipulate environmental cues, including studies of placebo and nocebo cognitions $[3,22,25,33,39,50,51]$.

Previously [4], we manipulated the level of certainty in the anticipation of a forthcoming pain stimulus using anticipation cues, demonstrating that "certain anticipation" modulates the perception of pain according to the content of the cue. Specifically, certain anticipation of a high-intensity laser pulse increased the perceived pain relative to uncertain anticipation. Furthermore, certain anticipation induced greater anticipatory activation in brain areas involved with semantic and prospective memory, whereas relative uncertainty was associated 
with greater anticipatory processing in an attention network. These results are suggestive of a probabilistic model of pain perception in which relative certainty increases the influence of expectations on perception and behavior, whereas greater uncertainty promotes attention and learning $[14,15]$.

In addition, more deep-seated beliefs/expectations about pain may arise from past experiences that could also affect the experience of pain and modify the extent to which environmental cues influence pain. However, to date, there has been no evidence of whether such "prior beliefs" bias how pain experience and neural activity are modulated by anticipatory cues. The probabilistic model of pain perception would suggest that confidence in prior beliefs (i.e. a greater certainty that they are true) would predict anticipatory influences on the experience of pain.

In this study we re-analyzed the data from our previous paper [4] in relation to an additional subjective measure of confidence. We hypothesized that we could measure confidence as a metacognitive process (i.e. a process that regulates cognition) that (1) is dissociable from the content of the expectation (2) uniquely influences pain perception and (3) is associated with activation of specific areas of the pain matrix.

\section{Materials and methods}

\subsection{Subjects}

Fifteen healthy, right-handed subjects participated in the study (14 female, 1 male; mean age $47 \pm 6.6$ ). Subjects gave informed written consent, and the study was approved by Oldham Local Research Ethics Committee.

\subsection{Experimental procedure}

The experimental procedure performed as previously described [4] is shown in Fig. 1. Laser heat stimuli of $150 \mathrm{~ms}$ duration and a beam diameter of $15 \mathrm{~mm}$ were applied to the dorsal surface of the subject's right forearm using a $\mathrm{CO}_{2}$ laser stimulator. These stimuli are known to selectively activate nociceptors related to $\mathrm{A} \delta$ and $\mathrm{C}$ fibres, without the need for skin contact [30]. Between stimuli, the laser was moved randomly over an area $3 \mathrm{~cm} \times 5 \mathrm{~cm}$ to avoid habituation, sensitization or skin damage. Subjects wore protective laser safety goggles during the experiment.

An initial psychophysics procedure was performed using a 0-10 pain rating scale, which was anchored such that level 4 indicated a pain threshold. A ramping procedure was repeated three times to determine the following three intensities of laser stimuli for each subject: level 3, level 5 and level 7, corresponding to "low" (non-painful), "medium" (just above pain threshold) and "high" (moderately painful) intensities of the laser heat stimuli, respectively. These semantic labels were used in the main part of the study to represent the three levels of heat intensity. Subjects, however, were not explicitly informed of this stratification of stimuli.

\subsection{Tuning the pain stimulus for reliability}

We then tested whether subjects were rating each level of intensity on the numerical scale as expected, and adjusted the intensity levels accordingly. This procedure was performed on every subject $(n=15)$ as follows: Subjects were given six pulses of each of the three intensity levels in a random sequence, totaling 18 pulses. After each pulse subjects rated the intensity of the stimulus on the $0-10$ pain rating scale. The average rating for each intensity level was then calculated. If the average rating deviated from the expected subjective intensity level $(3,5$ or 7$)$ by more than half a point on the scale, the laser energy was either increased or decreased accordingly. The procedure was then repeated as many times as necessary until the expected subjective intensity level was achieved.

\subsection{Subjective belieflexpectation of emotional distress}

Subjects were then asked to give their expected degree of emotional distress as a result of experiencing the laser pulses during the experiment. To do this, nine items were taken from the profile of mood states (POMS) scale, as had been done previously [44]. These were: sad, angry, discouraged, hopeless, hostile, irritable, tense, anxious and worried. Subjects were asked to rate the extent to which they expected to experience each of these emotions while experiencing the heat pulses during the experiment, by rating each one on a 5-point Likert scale from 0 ("not at all") to 4 ("very much"). The total score was taken as the expected degree of emotional distress. Subjects were asked to make these judgments partly on the basis of their own self-knowledge, partly on the basis of the information they had received about the experimental protocol (i.e. regarding the number of stimuli they would receive at each intensity over the course of the study), and partly based on their personal experience of the psychophysics procedure.

\subsection{Subjective confidence in belieflexpectation}

Then, subjects were asked to rate their confidence in this belief, that is, how strongly they believed their prediction to come true, using an 11-point numerical scale from 0 ("low confidence") to 10 ("high confidence"). Hence, this confidence measure was not a measure of the expected level of emotional distress itself, but rather a measure of how reliable they regarded their own belief/expectation to be. Here, confidence is a general measure of the perceived reliability of beliefs that related to the overall score on the POMS questionnaire, as opposed to individual items.

\subsection{Experimental stimuli and timings}

On each trial of the experiment (Fig. 1), a laser heat stimulus was delivered to the subject every $10 \mathrm{~s}$. Equal numbers of low, medium and high-intensity stimuli were delivered in a pre-determined randomized sequence, such that from the subject's perspective each of the stimuli could have occurred with equal chance on each trial. Laser stimuli were preceded by the appearance of an anticipatory visual cue, occurring $3 \mathrm{~s}$ prior to the laser stimulus, displayed on a computer monitor in front of the subject. The visual cue was a word ("low", "medium" or 


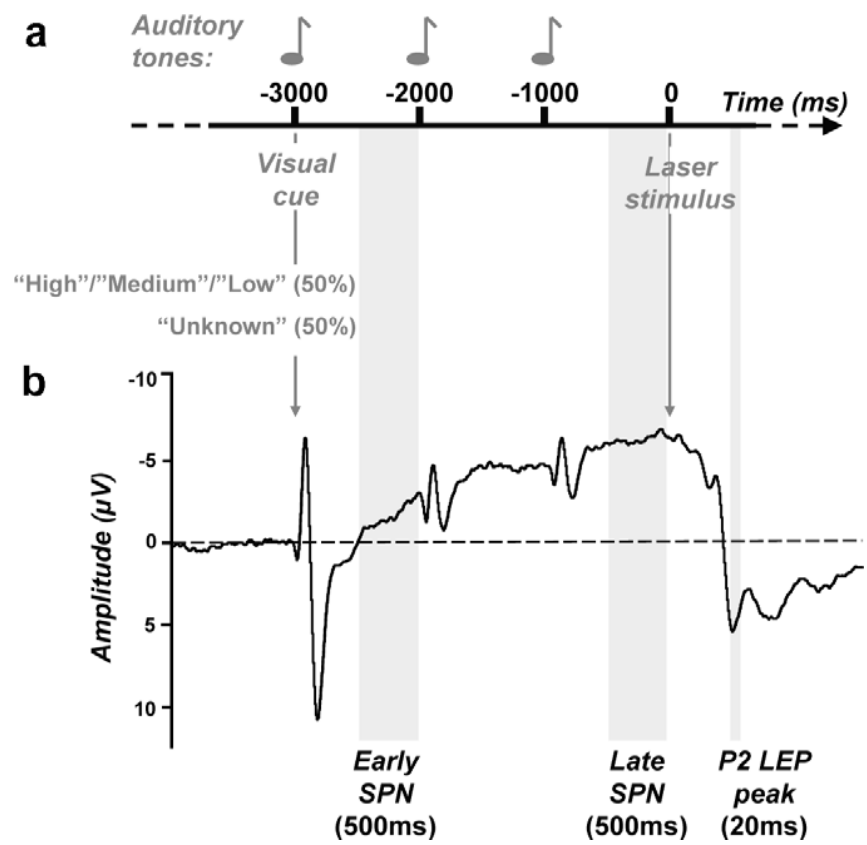

Fig. 1. (a) Experimental trial. "High", "medium" and "low"-intensity laser heat stimuli were preceded by certain or uncertain anticipatory cues that modulated expectations of forthcoming pain intensity. (b) The anticipation-evoked potential, or stimulus-preceding negativity (SPN) at electrode Cz. Visual cues induced a visual-evoked potential followed by an SPN, evident as a slow negative drift that increases in size until laser stimulus onset. Auditory-evoked potentials are also evident after -2000 and $-1000 \mathrm{~ms}$. Previously, we discovered that the SPN amplitude is modulated by the anticipated intensity of pain [4]. This is followed by a laser-evoked potential (LEP), of which the P2 peak is highlighted, which our previous work showed to correlate with the amplitude of the late SPN when expectations are certain [4]. Early ( -2500 to $-2000 \mathrm{~ms})$ and late ( -500 to $0 \mathrm{~ms})$ anticipatory stages, in addition to the P2 LEP peak (at each subject's individual latency), were extracted for source analysis (Fig. 3). (c) Topographic distribution of the early and late stages of the SPN and the P2 LEP peak.

"high") that faithfully predicted whether the following laser stimulus was to be of a low, medium or high-intensity. These "certain anticipation" trial types comprised $50 \%$ of the total trials. Certain anticipation cues were never violated. The other $50 \%$ were "uncertain anticipation" trial types in which an anticipatory cue reading "unknown" was followed by a high, medium or low intensity of heat stimulus with equal probability. In total, an equal number of laser stimuli at each intensity level were delivered to induce certain and uncertain anticipations. This constitutes a $3 \times 2$ factorial design (intensity $\times$ anticipation certainty). The onset of the visual cue occurred simultaneously with the first of three auditory tones applied using loud speakers that sounded at once per second until laser stimulus delivery, allowing for accurate prediction in the timing of the laser stimulus. The visual cue remained on the computer monitor during the trial. Three seconds after each laser pulse, an auditory tone of a different quality indicated to the subjects to verbally report the intensity of the experienced pain, using the $0-10$ pain scale. In total, 240 trials were presented in 4 blocks of 60 trials. The experiment lasted approximately $45 \mathrm{~min}$.

\subsection{Electroencephalographic (EEG) recordings}

EEG recordings were taken from 61 scalp electrodes placed according to an extended 10-20 system (Quik-Cap system, Neuroscan, Inc.). Bandpass filters were set at DC $-70 \mathrm{~Hz}$, with a sampling rate of $500 \mathrm{~Hz}$ and gain of 500 . A notch filter was set to $50 \mathrm{~Hz}$ to reduce electrical interference. Electrodes were referenced to the ipsilateral (right) earlobe, and recordings were also taken from the contralateral (left) earlobe for off-line conversion to linked-ears reference. The vertical and horizontal electro-oculograms (EOG) were measured for off-line reduction of blink and eye-movement artifacts.

\subsection{Behavioral data analysis}

The behavioral data were analyzed by calculating the percentage increase in subjective ratings of moderately painful laser heat stimuli ("high" intensity) in the certain relative to the uncertain condition. This was then correlated with the measure of confidence in expected emotional distress obtained prior to commencing the laser stimulation. Correlations were also made between the expected degree of emotional distress (i.e. not only the overall score, but also for each POMS item individually) and confidence in this expectation.

\subsection{EEG data analysis}

EEG data were analyzed using Neuroscan Edit 4.3. An ocular artifact reduction algorithm [42] was performed. The data were epoched into single trials of $5.5 \mathrm{~s}$ duration starting $1000 \mathrm{~ms}$ before the visual anticipation stimulus, including $3000 \mathrm{~ms}$ of anticipation, and ending $1500 \mathrm{~ms}$ seconds after the laser stimulus. Epochs were visually inspected for further ocular artifacts that had escaped automatic removal, and deleted if necessary. Linear trends over the whole epoch were then removed; the entire epoch was used to calculate the linear 
component in each epoch and channel separately, which was then removed from the data. The whole epoch was used in order to minimize removal of linear trends that may have specifically resulted from the anticipatory neural responses we were intending to measure. Each epoch was then baseline-corrected to the $500 \mathrm{~ms}$ interval preceding the visual predictive cue. The data were then averaged across the three stimulus intensities for each condition (certain and uncertain) separately. Data were referenced to the common average before proceeding further with data analysis, although ERP waveforms are presented according to the linked-ears reference.

Two $500 \mathrm{~ms}$ stages of the stimulus-preceding negativity (SPN), which constituted the anticipatory brain response, were analyzed. An "early" stage, at -2500 to $-2000 \mathrm{~ms}$ preceding the laser stimulus, was chosen as the earliest part of the anticipatory response that could be measured without interference from visual-evoked responses resulting from the anticipatory cue. A "late" stage, at -500 to $0 \mathrm{~ms}$ preceding the laser stimulus, was chosen to represent processes taking place in immediate preparation for the impending laser stimulus. The validity of this early vs. late distinction is drawn from the contingent negative variation $(\mathrm{CNV})$ literature $[13,20]$ and our previous work [4]. Lastly, we analyzed the P2 peak of the laser-evoked potential (LEP). Each subject's P2 peak latency was determined at electrode $\mathrm{Cz}$, where the topography of the P2 peak was maximal in the grand-averaged waveform. Data from two subjects were excluded from further analysis due to the lack of a P2 peak in the averaged waveform. A P2 peak was defined as a clearly visible positive-going potential that was (a) maximal within a latency of $\pm 200 \mathrm{~ms}$ of the grand average for the whole group, (b) increased in amplitude in a graded way by increasing laser pulse intensity. In the remaining subjects $(n=13)$, a $20 \mathrm{~ms}$ section of the $\mathrm{P} 2$ peak, $10 \mathrm{~ms}$ either side of the peak latency, was extracted for source analysis.

Cortical sources of the SPN were estimated with low-resolution electromagnetic tomography (LORETA), using the LORETA-KEY software [32]. The software uses a three-shell spherical head model registered to the Talairach anatomical brain atlas [45], although the electrode coordinates used were determined from a co-registration between spherical and realistic head geometries that create a best-fit model [46]. LORETA estimates ERP sources in grey matter volume to a 7$\mathrm{mm}^{3}$ grid resolution (2394 voxels in total) using the digitized MNI probability atlas [28]. Time-domain EEG files were converted to current density vector field magnitude using this technique. The resulting LORETA solutions were log-transformed at each pixel; this approximates LORETA solutions to a Gaussian distribution for parametric statistical analysis as previously demonstrated [24,27].

Although the precision of the LORETA solutions may have been partially compromised by the lack of information on subjects' individual anatomy, previous studies have reached satisfactory agreement between LORETA results and data obtained with more precise techniques, such as fMRI or subdural recordings $[31,41,47]$.

Once LORETA solutions have been generated in source space, the resulting images can be treated for statistical analysis in the same way as would fMRI data. LORETA solutions of intra-cerebral current density were converted to SPM image format using a modified version of LOR2SPM (http:// www.ihb.spb.ru/ pet_lab/L2S/L2SMain.htm). During this process LORETA solutions were intensity-normalized in order to eliminate subject-to-subject global variations. Statistical maps were then created using SPM5 (http://www.fil.ion.ucl.ac.uk/spm/software/spm5) running on Matlab 7.1 (Mathworks Inc.). For the anticipation (SPN) data, early and late stages were analyzed separately as follows: A second-level (group) ANOVA was constructed $(n=15)$ in order to explore the interaction between confidence in expected emotional distress and anticipatory cues (certain vs. uncertain). To do this, certain and uncertain conditions were contrasted after adding the confidence measure as a covariate in each condition separately. Results are reported at an uncorrected threshold of significance of $p<0.005$ (one-tailed), with a minimum of four contiguous voxels, within our areas of interest, that is, the primary areas of the pain matrix affected by expectation (cingulate, operculo-insular and inferior parietal cortices). For the P2 LEP peak data, a group ANOVA was constructed $(n=13)$ in order to explore the interaction between increased laser heat intensity (moderately painful vs. non-painful heat) and the anticipatory cues (certain vs. uncertain). Results are reported within our area of interest (right anterior insular cortex) at an uncorrected threshold of significance of $p<0.01$ (one-tailed), with a minimum of four contiguous voxels.

Statistically significant areas were analyzed as volumes of interest (VOIs) within SPM5. VOIs were extracted as the first eigenvector (i.e. the mean of the adjusted response after rejecting noise) of all voxels within a sphere of 7-mm radius, centered on the most significantly activated voxel, that were above the chosen statistical threshold. These values are relative to a whole-brain mean signal of 100 . Current density for each subject was then correlated and plotted against confidence in expected emotional distress and the percentage increase in pain perception separately $(n=15)$. Correlations reported are below the statistical threshold of $p<0.05$.

\section{Results}

\subsection{Behavioral results}

In comparing pain ratings for moderately painful ("high") laser heat stimuli between the certain and uncertain conditions, there was substantial variance across subjects in the direction and extent to which the certain cues relatively increased pain perception (Fig. 2). The percentage increase in the pain rating in the certain relative to the uncertain condition was significantly positively correlated with subjects' ratings of confidence in their prior beliefs about emotional distress $(r=0.65, p<0.01)$. However, there was no correlation between the percentage increase in pain experience and the expected degree of emotional distress (for either the total POMS score or for the individual items). There was also no correlation between the expected degree of emotional distress (for total and individual POMS item scores) and confidence in that prediction. Together, these results indicate that confidence predicts anticipatory cue-induced modulation of pain perception independently of the degree of expected emotional distress. 

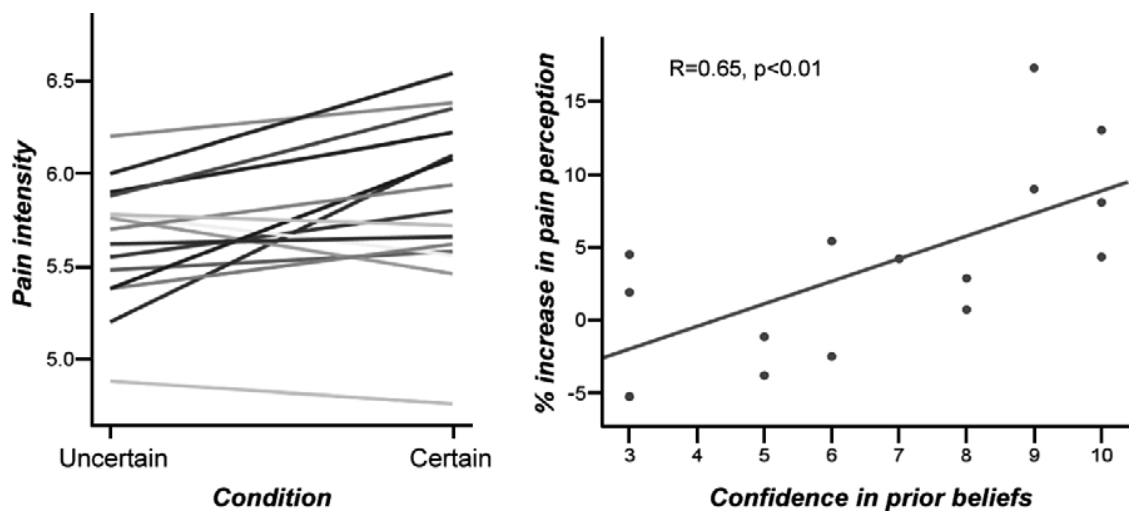

Fig. 2. Behavioral data. (a) Subjective pain experience was rated using a $0-10$ numeric scale $(4=$ pain threshold) in response to moderately painful ("high" intensity) laser heat stimuli, shown here for each of the certain and uncertain conditions (one line per subject). Cues that accurately predicted pain intensity (certain condition) modified pain ratings relative to the uncertain condition. (b) The percentage increase in pain perception in the certain condition, relative to the uncertain condition, across subjects shows a significant positive correlation with subjects' ratings of confidence in their prior expectations of emotional distress.

\subsection{EEG results}

In our previous analysis of this dataset, uncertain anticipation was associated with greater anticipatory neural activity in brain regions associated with attention (left dorsolateral prefrontal, bilateral inferior parietal and posterior cingulate cortices) whereas certain anticipation was associated with greater activity in memory areas (inferior temporal and prefrontal cortices, hippocampus and right frontal pole) [4]. In the current analysis, we determined whether areas of the pain matrix were associated with the interaction between confidence in expected emotional distress and the anticipatory cues (certain anticipation minus uncertain anticipation contrast). During "early" anticipation of pain (-2500 to $-2000 \mathrm{~ms}$ pre-laser stimulus, (Fig. 3), confidence in prior expectations interacted positively with the anticipatory cues (certain vs. uncertain) in right anterior insular cortex, and negatively with the anticipatory cues (certain vs. uncertain) in right inferior parietal cortex and midcingulate cortex (Table 1). There were no significant interactions found during late anticipation.

Volume of interest (VOI) analyses of these brain areas (see Section 2 and Fig. 3) showed that this interaction was driven by a positive correlation between confidence in expected emotional distress and activity in right anterior insular cortex in the certain condition $(r=0.73$, $p<0.01)$ but not the uncertain condition $(r=0.18)$ during early anticipation. Conversely, in the certain condition but not the uncertain condition, confidence in expected emotional distress negatively correlated with activity in VOIs representing the right inferior parietal cortex (certain condition: $r=-0.80, p<0.01$; uncertain condition: $r=0.07$ ) and midcingulate cortex (certain condition: $r=-0.79, p<0.01$, uncertain condition: $r=-0.06)$ during early anticipation. Confidence in expected emotional distress, therefore, modified the extent to which anticipatory cues modulated activity in these key regions of the pain matrix.

The percentage increase in the experienced intensity of moderately painful stimuli resulting from certain vs. uncertain anticipatory cues was also found to correlate with VOIs during early anticipation in the certain condition (Fig. 3). A positive correlation was found with activity in right anterior insula $(r=0.61, p<0.05)$ and a negative correlation with activity in right inferior parietal cortex $(r=-0.62, p<0.05)$. There was also a negative correlation with activity in the midcingulate cortex $(r=-0.46)$ that did not reach statistical significance. This suggests that activity in the right anterior insular and inferior parietal cortices during pain anticipation may mediate the effects of anticipatory cues on pain perception. To test this hypothesis, we analyzed source-localized activity during the $\mathrm{P} 2$ peak of the laser-evoked potential (LEP) in order to find neural substrates associated with the interaction between increased laser heat stimulus intensity (i.e. moderately painful vs. non-painful) and the certain vs. uncertain anticipatory cues (Fig. 3). A positive interaction was identified in the right anterior insular cortex (Table 1), suggesting a role for this region in the modulation of pain processing by expectation. Right inferior parietal or midcingulate cortex activity, however, was not associated with this interaction.

\section{Discussion}

In this study we assessed subjects' prior beliefs that experimental pain would cause them emotional distress, and obtained ratings of their confidence in those beliefs. We predicted that confidence would interact with anticipatory pain processing in areas of the pain matrix known to be involved with expectation and aversive conditioning, such as the cingulate, operculo-insular and inferior parietal cortices $[5,7,25,36,43,50]$. The 
a Interaction between confidence in prior beliefs and anticipatory cues

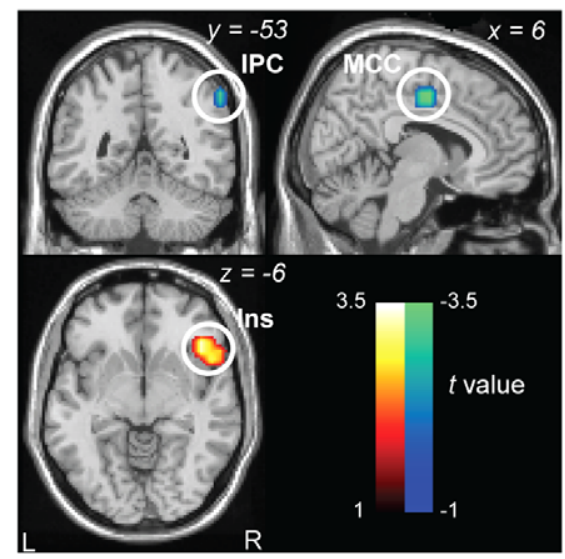

d Interaction between intensity of laser heat stimulus and anticipatory cues

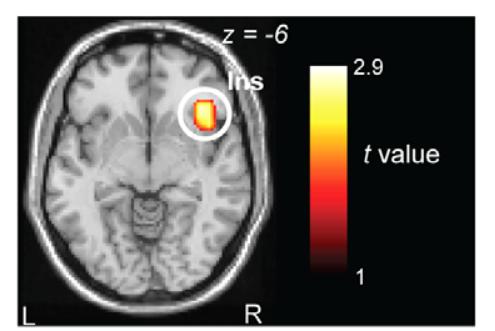

b Effects of confidence during certain anticipation
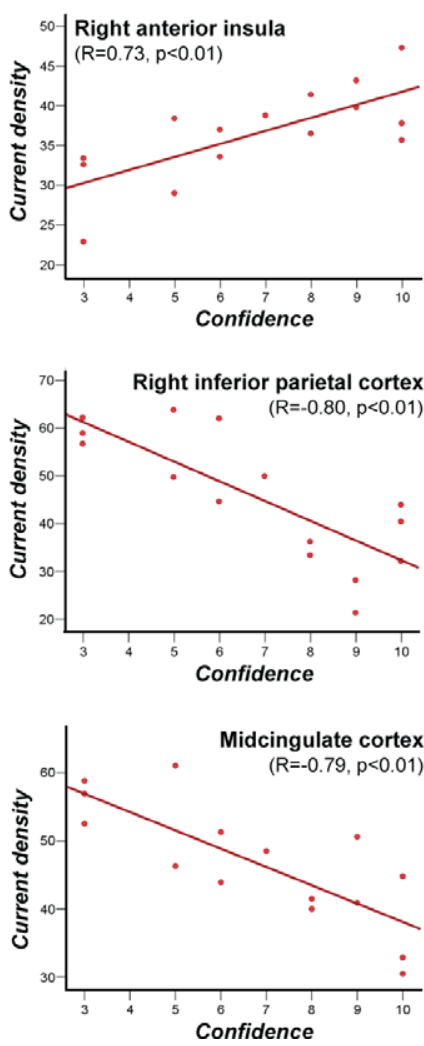

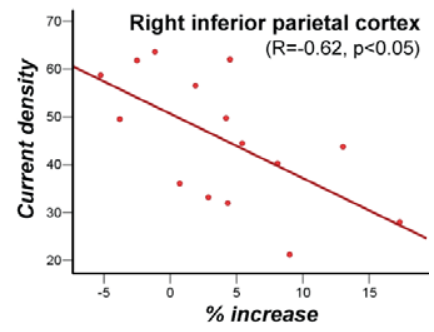

\section{Anticipatory predictors of increased pain perception}

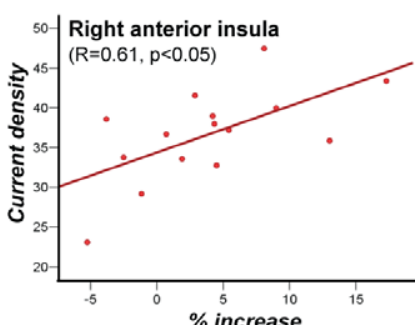

Fig. 3. Variation of current density with behavioral data. (a) During "early" anticipation of pain, confidence in prior expectations of emotional distress interacted positively with certain vs. uncertain anticipation cues in right anterior insula cortex (Ins), and negatively with certain vs. uncertain cues in right inferior parietal cortex (IPC) and midcingulate cortex (MCC). (b) Confidence in prior expectations of emotional distress positively correlated with activity in right anterior insula cortex during certain anticipation (but not uncertain anticipation), and negatively correlated with activity in right inferior parietal and midcingulate cortex. (c) The same regions of right anterior insular and inferior parietal cortices during certain anticipation show positive and negative correlations, respectively, with the increased pain experience resulting from anticipation cues (certain vs. uncertain). (d) During the laser-evoked potential (LEP, P2 peak), increased laser heat stimulus intensity (moderately painful vs. non-painful) interacted with the anticipation cues (certain vs. uncertain) in right anterior insula cortex.

results illustrate three key findings. Firstly, confidence in prior beliefs about emotional distress predicts the extent to which pain experience can be experimentally modulated using anticipatory cues. Secondly, a key role is demonstrated for the right anterior insula in the influ- ence of prior beliefs on anticipatory modulation of pain. Thirdly, right anterior insular and inferior parietal cortices adopt reciprocal roles during anticipation of pain, with opposite influences on the modulation of pain experience by anticipatory cues.

Table 1

Brain regions showing variations in current density with behavioral data and task conditions

\begin{tabular}{lllll}
\hline Brain region & Area & MNI coordinates & $t$ Value & $z$ Value (uncorr)
\end{tabular}

Early anticipation: correlation with confidence in prior beliefs

Positive correlation

Anterior insular cortex

$\mathrm{R} \quad 13$

39

0.001

Negative correlation

Inferior parietal cortex

$\mathrm{R} \quad 40$

53

53

Midcingulate cortex

L 31

$-10$

$-11$

43

3.18

0.002

P2 LEP peak: interaction between stimulus intensity and anticipation cues

Positive interaction
Anterior insular cortex

$\begin{array}{llll}\mathrm{L} & 13 & 32 & 24\end{array}$

$24-6$

4.31

0.000

Uncorr, uncorrected $p$ values; Area, Brodmann's area. 


\subsection{The effects of confidence on anticipatory modulation of pain ratings}

A correlation was found between confidence in reported beliefs about emotional distress and the extent to which pain experience was modulated by anticipatory cues. The subjects' judgments of confidence in their predicted level of emotional distress arose from the perceived reliability of their belief. According to contemporary (probabilistic) theories of perception, greater reliability of a predictor increases its influence on perception and behavior $[18,23,53]$. Previous work has manipulated this reliability by varying the content of the predictive information, thereby modifying its predictive accuracy $[4,35]$. By contrast, the confidence measure does not reflect variation in the objective accuracy of the predictive information provided, but rather reflects a subjective bias in the extent to which predictive information is regarded as accurate. For this reason we regard confidence as indexing a "metacognitive" function (perhaps relating to a personality trait) that modulates the extent of top-down influences on pain. Given that expected emotional distress did not relate to pain modulation or anticipatory EEG responses, it may be that the specific object of the confidence rating is less important than the tendency of subjects to regard predictions as reliable. However, the aforementioned probabilistic theories of perception imply an interaction between confidence and expectation. Unfortunately we did not have the statistical power in the current sample to test this interaction, but this is an important direction for future studies.

We suggest that the influence of the anticipatory cues on pain experience in this study will have arisen from the subjectively perceived reliability of the certain anticipatory cues in predicting pain. This is because although certain anticipation cues accurately predicted the delivered laser intensity, there was no guarantee that subjects would regard these cues as accurate. Our results, showing a relationship between the influence of anticipation cues on pain and confidence in prior beliefs, are best explained by the common influence of a metacognitive process (indexed by confidence) on expectations generated from external (i.e. anticipation cues) and internal (i.e. prior beliefs) sources of knowledge. However, it cannot be determined from these results whether or not this metacognitive process modulates top-down effects on other phenomena unrelated to either pain or the other aspects of the experimental context.

\subsection{Neural mechanisms mediating the effects of prior beliefs on pain}

Although our behavioral data cannot further characterize those processes that give rise to confidence judgments, the EEG results suggest a key role for the right anterior insula in mediating the interaction between the confidence in prior beliefs, anticipatory cues and pain. Firstly, early activation of right anterior insula during pain anticipation, when subjects knew the intensity of the forthcoming stimulus, correlated with confidence in prior beliefs. Secondly, the correlation of certain anticipatory activity with the subsequent extent of pain modulation (shown behaviorally) by anticipatory cues suggests a possible role for right anterior insula in mediating the influence of expectations on subsequent pain experience. Lastly, nociceptive processing in the right anterior insula (during the P2 LEP peak) was increased by knowledge of the forthcoming intensity for painful vs. non-painful stimuli. Together, these results highlight a key role for right anterior insula in integrating prior information with subsequent perception of pain, both in terms of subjective confidence in one's beliefs and with respect to the perceived reliability of external cues.

The anterior insula has a well-established role in pain and its anticipation [7,11], and several key findings have been noted. Firstly, activity in this area shows a greater association with subjective pain ratings than actual increases in temperature [12]; secondly, anticipatory activity here has been seen immediately preceding a predictable pain stimulus $[8,36,43]$ which overlaps with subsequent pain-related activity [25]; and thirdly, anticipatory brain activity in this area has already been shown to predict subjective pain perception [37]. The present data suggest that right anterior insula may have a specific role in mediating the effects of the perceived reliability of beliefs and expectations on subsequent pain experience. Our data showing early-stage modulation of right anterior insula may correspond to motivational processes that typically occur at the time when expectation is first generated [43].

It may appear surprising that our data did not reveal any interactions between knowledge of the forthcoming pain intensity and the actual stimulus intensity in the posterior insula during the P2 LEP peak. Posterior insula is known to be involved in pain intensity coding [9] and the generation of LEPs [19], and has been previously shown to be modulated by expectation [40]. Our results showing activation of anterior insular cortex ipsilateral to the stimulated arm during anticipation and pain may indicate that more anterior regions of the insular cortex are specialized towards mediating top-down effects on pain that are less dependent on laterality and therefore sensory afferent input. Further work will be required to ascertain whether the same hemispheric pattern is obtained after stimulation of the left forearm; this would suggest that right anterior insula mediates top-down effects on pain regardless of the laterality of stimulation.

Our results also showed that early anticipatory processing in right inferior parietal and midcingulate corti- 
ces was negatively associated with confidence in prior beliefs about emotional distress. One possible explanation for this arises from consideration of attentional aspects of perception. Specifically, if prior beliefs are held with greater certainty, there are fewer requirements to allocate attentional resources to the ascending nociceptive input (a concept formalized in theoretical models of attention $[15,53]$. Thus, one would expect increases in activity in areas associated with utilizing prior information (i.e. the right anterior insula) to be coupled with decreased activity in areas involved in attention and sensory perception. A large body of evidence corroborates this explanation of our data, implicating a key role for right inferior parietal and midcingulate cortices (which are heavily interconnected anatomically $[1,49]$ in attention, particularly with respect to pain $[2,6,10,16,34,38,40]$. Furthermore, anatomical connections have been demonstrated in monkeys between the cingulate and insula cortices that may allow for reciprocal activation patterns during pain expectancy [49]. Midcingulate and inferior parietal cortices may therefore form part of a network that is responsive to pain anticipation (as expressed in insular cortex) and acts to maintain sensory influences on perception when prior expectations are less certain.

\subsection{Clinical implications}

From a clinical perspective, the bias induced by confidence in prior beliefs may offer insight into the role of pain expectancy in the pathophysiology of chronic pain and cognitive interventional approaches to its treatment. It is worth mentioning here that these data are mostly representative of the female brain, as only one subject was male. This may have relevance to unexplained pain conditions such as fibromyalgia in which female gender is a significant risk factor [52] and in which distressing beliefs about pain may play a key role in the symptomology [21]. However, without a formal comparison of male and female subject data we cannot draw conclusions about the relationship of female gender to the results. This is an important direction for future studies.

The results suggest that the degree of expected emotional distress from pain did not predict pain modulation by anticipation cues, and there was a lack of correlation between the degree of expected emotional distress and confidence in that prediction. This suggests a functional dissociation between the belief and its perceived reliability. Although our results suggest that only the latter influences pain ratings, theoretical considerations $[18,23,53]$ imply an interaction between the belief and its perceived reliability. This dissociation is relevant to cognitive-behavioral treatments for chronic pain and related affective disorders such as anxiety and depression that aim to modify the extent to which beliefs influence behavior [29]. In particular, cognitive-behavioral treat- ments may be improved by teaching patients core skills in questioning their beliefs as a method to reduce the extent to which they are held with certainty.

\section{Acknowledgements}

There are no financial or other relationships that might lead to a conflict of interest in publishing this article. This work was funded by the University of Manchester and the Arthritis Research Campaign. We thank Professor Steve Williams and Dr. Lloyd Gregory at the University of Manchester for their input and support of this work.

\section{References}

[1] Baleydier C, Mauguiere F. The duality of the cingulate gyrus in monkey. Neuroanatomical study and functional hypothesis. Brain 1980;103:525-54.

[2] Behrmann M, Geng JJ, Shomstein S. Parietal cortex and attention. Curr Opin Neurobiol 2004;14:212-7.

[3] Benedetti F, Amanzio M. The neurobiology of placebo analgesia: from endogenous opioids to cholecystokinin. Prog Neurobiol 1997;52:109-25.

[4] Brown CA, Seymour B, Boyle Y, El-Deredy W, Jones AKP. Modulation of pain perception by expectation and uncertainty: behavioral characteristics and anticipatory neural correlates. Pain 2008;135:240-50.

[5] Buchel C, Dolan RJ. Classical fear conditioning in functional neuroimaging. Curr Opin Neurobiol 2000;10:219-23.

[6] Buffington AL, Hanlon CA, McKeown MJ. Acute and persistent pain modulation of attention-related anterior cingulate fMRI activations. Pain 2005;113:172-84.

[7] Calder AJ, Lawrence AD, Young AW. Neuropsychology of fear and loathing. Nat Rev Neurosci 2001;2:352-63.

[8] Chua P, Krams M, Toni I, Passingham R, Dolan R. A functional anatomy of anticipatory anxiety. Neuroimage 1999;9:563-71.

[9] Coghill RC, Sang CN, Maisog JM, Iadarola MJ. Pain intensity processing within the human brain: a bilateral, distributed mechanism. J Neurophysiol 1999;82:1934-43.

[10] Coull JT, Nobre AC. Where and when to pay attention: the neural systems for directing attention to spatial locations and to time intervals as revealed by both PET and fMRI. J Neurosci 1998;18:7426-35

[11] Craig AD. How do you feel? Interoception: the sense of the physiological condition of the body. Nat Rev Neurosci 2002;3:655-66.

[12] Craig AD, Chen K, Bandy D, Reiman EM. Thermosensory activation of insular cortex. Nat Neurosci 2000;3:184-90.

[13] Cui RQ, Egkher A, Huter D, Lang W, Lindinger G, Deecke L. High resolution spatiotemporal analysis of the contingent negative variation in simple or complex motor tasks and a non-motor task. Clin Neurophysiol 2000;111:1847-59.

[14] Dayan P, Abbott LF. Theoretical neuroscience: computational and mathematical modeling of neural systems. MIT Press; 2001.

[15] Dayan P, Kakade S, Montague PR. Learning and selective attention. Nat Neurosci 2000;3(Suppl.):1218-23.

[16] Fan J, McCandliss BD, Fossella J, Flombaum JI, Posner MI. The activation of attentional networks. Neuroimage 2005;26:471-9.

[17] Fendt M, Fanselow MS. The neuroanatomical and neurochemical basis of conditioned fear. Neurosci Biobehav Rev 1999;23:743-60. 
[18] Friston K. Learning and inference in the brain. Neural Netw 2003; 16:1325-52.

[19] Garcia-Larrea L, Frot M, Valeriani M. Brain generators of laserevoked potentials: from dipoles to functional significance. Neurophysiol Clin 2003;33:279-92.

[20] Gomez CM, Marco J, Grau C. Preparatory visuo-motor cortical network of the contingent negative variation estimated by current density. Neuroimage 2003;20:216-24.

[21] Hassett A, Cone J, Patella S, Sigal L. The role of catastrophizing in the pain and depression of women with fibromyalgia syndrome. Arthritis Rheum 2000;43:2493-500.

[22] Keltner JR, Furst A, Fan C, Redfern R, Inglis B, Fields HL. Isolating the modulatory effect of expectation on pain transmission: a functional magnetic resonance imaging study. J Neurosci 2006;26:4437-43.

[23] Kersten D, Yuille A. Bayesian models of object perception. Curr Opin Neurobiol 2003;13:150-8.

[24] Kiebel SJ, Tallon-Baudry C, Friston KJ. Parametric analysis of oscillatory activity as measured with EEG/MEG. Hum Brain Mapp 2005;26:170-7.

[25] Koyama T, McHaffie JG, Laurienti PJ, Coghill RC. The subjective experience of pain: where expectations become reality. Proc Natl Acad Sci USA 2005;102:12950-5.

[26] LeDoux J. The emotional brain, fear, and the amygdala. Cell Mol Neurobiol 2003;23:727-38.

[27] Liu Y, Perfetti CA. The time course of brain activity in reading English and Chinese: an ERP study of Chinese bilinguals. Hum Brain Mapp 2003;18:167-75.

[28] Mazziotta J, Toga A, Evans A, Fox P, Lancaster J, Zilles K, et al. A four-dimensional probabilistic atlas of the human brain. $\mathbf{J}$ Am Med Inform Assoc 2001;8:401-30.

[29] McCracken LM. Contextual cognitive behavioral therapy for chronic pain, vol. 33. IASP Press; 2005.

[30] Meyer RA, Walker RE, Mountcastle Jr VB. A laser stimulator for the study of cutaneous thermal and pain sensations. IEEE Trans Biomed Eng 1976;23:54-60.

[31] Mulert C, Jager L, Schmitt R, Bussfeld P, Pogarell O, Moller HJ, et al. Integration of fMRI and simultaneous EEG: towards a comprehensive understanding of localization and time-course of brain activity in target detection. Neuroimage 2004;22:83-94.

[32] Pascual-Marqui RD, Esslen M, Kochi K, Lehmann D. Functional imaging with low-resolution brain electromagnetic tomography (LORETA): a review. Methods Find Exp Clin Pharmacol 2002;24(Suppl. C):91-5.

[33] Petrovic P, Kalso E, Petersson KM, Ingvar M. Placebo and opioid analgesia - imaging a shared neuronal network. Science 2002;295:1737-40.

[34] Peyron R, Garcia-Larrea L, Gregoire MC, Costes N, Convers P, Lavenne $\mathrm{F}$, et al. Haemodynamic brain responses to acute pain in humans: sensory and attentional networks. Brain 1999;122(Pt 9):1765-80.

[35] Ploghaus A, Narain C, Beckmann CF, Clare S, Bantick S, Wise R, et al. Exacerbation of pain by anxiety is associated with activity in a hippocampal network. Journal of Neuroscience 2001;21:9896-903.
[36] Ploghaus A, Tracey I, Gati JS, Clare S, Menon RS, Matthews PM, et al. Dissociating pain from its anticipation in the human brain. Science 1999;284:1979-81.

[37] Porro CA, Baraldi P, Pagnoni G, Serafini M, Facchin P, Maieron $\mathrm{M}$, et al. Does anticipation of pain affect cortical nociceptive systems? J Neurosci 2002;22:3206-14.

[38] Posner MI, Rothbart MK. Attention, self-regulation and consciousness. Philos Trans R Soc Lond B Biol Sci 1998;353:1915-27.

[39] Price DD. Psychological mechanisms of pain and analgesia, vol. 15. IASP Press; 1999.

[40] Sawamoto N, Honda M, Okada T, Hanakawa T, Kanda M, Fukuyama H, et al. Expectation of pain enhances responses to nonpainful somatosensory stimulation in the anterior cingulate cortex and parietal operculum/posterior insula: an event-related functional magnetic resonance imaging study. J Neurosci 2000;20:7438-45.

[41] Seeck M, Lazeyras F, Michel CM, Blanke O, Gericke CA, Ives J, et al. Non-invasive epileptic focus localization using EEGtriggered functional MRI and electromagnetic tomography. Electroencephalogr Clin Neurophysiol 1998;106:508-12.

[42] Semlitsch HV, Anderer P, Schuster P, Presslich O. A solution for reliable and valid reduction of ocular artifacts, applied to the P300 ERP. Psychophysiology 1986;23:695-703.

[43] Seymour B, O'Doherty JP, Dayan P, Koltzenburg M, Jones AK, Dolan RJ, et al. Temporal difference models describe higher-order learning in humans. Nature 2004;429:664-7.

[44] Sullivan MJ, Rodgers WM, Kirsch I. Catastrophizing, depression and expectancies for pain and emotional distress. Pain 2001;91:147-54.

[45] Talairach J, Tournoux P. Co-planar stereotaxic atlas of the human brain. New York: Thieme; 1988.

[46] Towle VL, Bolanos J, Suarez D, Tan K, Grzeszczuk R, Levin DN, et al. The spatial location of EEG electrodes: locating the bestfitting sphere relative to cortical anatomy. Electroencephalogr Clin Neurophysiol 1993;86:1-6.

[47] Vitacco D, Brandeis D, Pascual-Marqui R, Martin E. Correspondence of event-related potential tomography and functional magnetic resonance imaging during language processing. Hum Brain Mapp 2002;17:4-12.

[48] Vlaeyen JW, Linton SJ. Fear-avoidance and its consequences in chronic musculoskeletal pain: a state of the art. Pain 2000;85:317-32.

[49] Vogt BA, Pandya DN. Cingulate cortex of the rhesus monkey: II. Cortical afferents. J Comp Neurol 1987;262:271-89.

[50] Wager TD, Rilling JK, Smith EE, Sokolik A, Casey KL, Davidson RJ, et al. Placebo-induced changes in FMRI in the anticipation and experience of pain. Science 2004;303:1162-7.

[51] Watson A, El-Deredy W, Vogt BA, Jones AK. Placebo analgesia is not due to compliance or habituation: EEG and behavioural evidence. Neuroreport 2007;18:771-5.

[52] Wolfe F, Ross K, Anderson J, Russell IJ, Hebert L. The prevalence and characteristics of fibromyalgia in the general population. Arthritis Rheum 1995;38:19-28.

[53] Yu AJ, Dayan P. Uncertainty, neuromodulation, and attention. Neuron 2005;46:681-92. 